\title{
Cytoreductive Surgery and Hyperthermic Intraperitoneal Chemotherapy (HIPEC): The Need for Accessible Data in Real Time
}

\author{
Glen C. Balch, $\mathrm{MD}^{1}$ and David L. Bartlett, $\mathrm{MD}^{2}$ \\ ${ }^{1}$ University of Texas Southwestern Medical Center, Dallas, TX; ${ }^{2}$ University of Pittsburgh, Pittsburgh, PA
}

Many new treatments for cancer have been developed based on successful anecdotes, single-institution retrospective studies, and small, poorly powered and controlled randomized trials, especially in the surgical world, in which completion of randomized trials has been challenging. Although these treatments have sometimes cured patients who had no hope, and prolonged high-quality survival for patients sentenced to weeks of life, they often are applied beyond their initial indications as physicians strive to offer hope to desperate patients. In these cases of novel, controversial, or "off-label" treatments, it is imperative that we record and maintain accurate data.

To date, many investigators have relied on data managers, who are not well trained to record data, which may not be accurate in a semi-prospective format. Years may pass before these data are analyzed and the results written and published. More years may elapse before the results are digested by the medical community, and even more years may be required for practice to change. In the end, we still may make the wrong decisions because the data may not be presented completely, or we may doubt the validity of outdated information.

The financial world or even the sports world could not survive on the aforementioned model. Instead, they have real-time data at their fingertips to assess each unique situation and make highly educated decisions based on current data.

The medical field remains far behind. This issue is highlighted in the manuscript by Simkens et al. ${ }^{1}$. entitled

(C) Society of Surgical Oncology 2015

First Received: 15 July 2015;

Published Online: 12 August 2015

D. L. Bartlett, MD

e-mail: bartdl@upmc.edu
"Treatment-Related Mortality After Cytoreductive Surgery and HIPEC in Patients With Colorectal Peritoneal Carcinomatosis Is Underestimated by Conventional Parameters." In their study, the authors discovered that reported mortality rates for hyperthermic intraperitoneal chemotherapy (HIPEC) may inaccurately reflect the actual mortality rates.

The authors examined 245 consecutive cases of complete cytoreduction (no gross residual disease) and HIPEC for colorectal cancer and looked in depth at the cause of death during the first year. The classic 30-day mortality rate was only $1.6 \%$, which is considered a good outcome for such a difficult disease. In general, when physicians publish their data, they desire to make the data look good, so the value of $1.6 \%$ is what often would be published from such a series. Clinicians managing patients with peritoneal carcinomatosis would use that number to make treatment decisions. However, when the authors examined treatment-related death during the first year, the actual number changed to $4.9 \%$. In addition, another $7.9 \%$ of the patients died during the first year due to rapid recurrence. Although this difference may not change the treatment recommendations, it should be considered and should change the discussion with the patient.

The authors noted that those with a higher burden of disease, requiring a larger, more complicated operation, had a statistically higher likelihood of dying (of complications or early recurrence) during the first year even though the study was controlled for complete gross resection. Unfortunately, the manuscript does not give data on the 1-year mortality for those with a high burden of disease. Because the overall 1-year mortality rate was $13.9 \%$, it would not be unreasonable to imagine that the rate for those with a high burden of disease could be in the range of $25 \%$ or even higher. This still may not change the recommendation for patients with no other options, but it 
should certainly influence the decision making and have an impact on the expectation of the patients. What if findings eventually show a 1-year mortality rate of $80 \%$ for the last 100 patients older than 70 years with high-volume disease who failed chemotherapy and have a BRAF mutation? We should not need to wait for years to have that data to consider for patient management decisions.

This experience is not unique to the data from Simkins et al. ${ }^{1}$ We recently reported a 60 -day mortality rate of $1.9 \%$ for 370 cases of CRS/HIPEC. ${ }^{2}$ A rapid analysis of our database comprising 1314 patients undergoing CRS/HIPEC (assessed for the purpose of this editorial) shows that $8 \%$ died within 6 months after treatment (unpublished). We had not previously appreciated that difference and have not had real-time access to our own data for patient decision making. This experience also is not unique to surgical oncology. The reported 90-day mortality rate for hip replacement surgery is $0.65 \%$. $^{3}$ For patients older than 60 years receiving hemodialysis, that 90-day mortality rate increases to $17.3 \% .^{4}$ Although we recognize the factors that increase risk, we do not know the magnitude of that increased risk in real time for intelligent discussions with our patients.

One method of enhancing access to data for information on off-label drug use is through a patient-centered, online research platform into which thousands of patients have entered and accessed organized data on off-label use of medical products. ${ }^{5}$ This approach uses subjective patient data entered online to track the results of off-label treatment for diseases such as multiple sclerosis, Parkinson's disease, mood conditions, fibromyalgia/chronic fatigue syndrome, and amyotrophic lateral sclerosis. Taking advantage of existing electronic medical records and novel online applications to supply more objective data in a userfriendly way to physicians and patients should be feasible. The key is entry of accurate data and existence of a manageable interface for real-time access.

It is a shame that a practicing physician does not currently have access to accurate, complete data to mine in real time to help make decisions on patients. In contrast, a manager for a baseball team can access data on the last 15 times his batter faced a left-handed pitcher in late innings with runners in scoring position and can decide whether he should substitute a pinch hitter. He also can access data on the pinch hitter and how many times he faced this particular pitcher during his 15-year career and what were his batting average, strike-out rate, on-base-percentage and double-play percentage. The manager then can make an educated decision based on real-time statistics.

On the other hand, if a surgical oncologist has a patient come to the office with high-volume peritoneal carcinomatosis from colorectal cancer, he or she must review manuscripts from generic data at least 5 years old that likely is flawed and incomplete and then make a very significant decision for that patient's life. The oncologist may want to know the 1-year mortality rate for patients with high-volume disease. He or she may want to filter the data for patients younger than 40 years with high-volume disease or patients younger than 40 years with a good response to chemotherapy and high-volume disease. The clinician should have access to all the available data in real time to make an educated decision about the best treatment option for that particular patient. Furthermore, the patient also should have access to that data so that if the patient wants to be involved in the decision-making process, he or she has accurate data to consider.

The manuscript by Simkens et al. ${ }^{1}$ is a step in the right direction, but a small step. It is only an example of how the data normally provided in manuscripts are incomplete and may influence our decision making in a flawed manner. We have long recognized the flaws in retrospective data, single-institution studies, and anecdotal case reports. This has driven the field toward trusting only randomized trials. This process is still flawed, however, and requires an enormous amount of time for planning, funding, accruing, analyzing, reporting, reading, digesting, and changing practice in a field that is more dynamic and individualized than baseball. Would it be more useful for the baseball manager to run a randomized trial on left- versus right-handed batters against left-handed pitchers or to have access to data on what the pitcher's results were the last 30 times he threw against left-handed batters with runners in scoring position during night games at his home field.

As bioinformatics and accurate electronic records improve, the goal of real-time access to data and assessment of risk and outcomes is possible and necessary. For treatments considered controversial or "off label," it should be mandatory that accurate outcome data be tracked and recorded by any physician using that treatment, and the data should be accessible in real time to physicians and patients. Although this may seem like a ridiculous goal, we should consider what the Oakland Athletics accomplished in 2002. Using a data-driven, analytic, "sabermetric" approach to baseball, they were able to win their division with the third lowest payroll in the major leagues and transform the way baseball is managed. ${ }^{6}$ Access to medical treatment results and real-time use of data for medical decision making need to be similarly transformed.

CONFLICT OF INTEREST The author declares no conflicts of interest.

\section{REFERENCES}

1. Simkens GA, van Oudheusden TR, Braam HJ, Luyer MD, Wiezer MJ, van Ramshorst B, Nienhuijs SW, de Hingh IH. Treatmentrelated mortality after cytoreductive surgery and HIPEC in patients 
with colorectal peritoneal carcinomatosis is underestimated by conventional parameters. Ann Surg Oncol. 2015. doi:10.1245/ s10434-015-4699-5.

2. Polanco PM, Ding Y, Knox JM, Ramalingam L, Jones H, Hogg ME, Zureikat AH, Holtzman MP, Pingpank J, Ahrendt S, Zeh HJ, Bartlett DL, Choudry HA. Institutional learning curve of cytoreductive surgery and hyperthermic intraperitoneal chemoperfusion for peritoneal malignancies. Ann Surg Oncol. 2015;22:1673-9.

3. Berstock JR, Beswick AD, Lenguerrand E, Whitehouse MR, Blom AW. Mortality after total hip replacement surgery: a systematic review. Bone Joint Res. 2014;3:175-182. PMCID: PMC4054013.
4. Chien-Fu Lin J, LiangW-M. Mortality and complications after hip fracture among elderly patients undergoing hemodialysis. $B M C$ Nephrol. 2015;16:100. PMCID: PMC4492013.

5. Frost J, Okun S, Vaughan T, Heywood J, Wicks P. Patient-reported outcomes as a source of evidence in off-label prescribing: analysis of data from patients like me. J Med Internet Res. 2011;13:e6. PMCID: PMC3221356.

6. Lewis M. Moneyball: The Art of Winning an Unfair Game. 1st ed. W.W. Norton, New York, 2003. 